\title{
CYP2D6 in the Metabolism of Opioids for Mild to Moderate Pain
}

\author{
Wojciech Leppert
}

Chair and Department of Palliative Medicine, Poznan University of Medical Sciences, Poznan, Poland

\section{Key Words}

Analgesics $\cdot$ Cancer pain $\cdot$ CYP2D6 $\cdot$ Opioids $\cdot$ Pain

treatment $\cdot$ Weak opioids

\begin{abstract}
In most cancer patients, pain is successfully treated with pharmacological measures using opioid analgesics for moderate to severe pain (strong opioids) alone or in combination with adjuvant analgesics (coanalgesics). Opioids for mild to moderate pain (weak opioids) are usually recommended in the treatment of cancer pain of mild to moderate intensity. There is a debate whether the second step of the WHO analgesic ladder comprising weak opioids such as tramadol, codeine and dihydrocodeine is still needed for the treatment of cancer and chronic pain since low doses of strong opioids show similar efficacy. However, many patients with mild, moderate and in some cases strong pain intensity are still successfully treated with weak opioids. All these drugs are metabolized through CYP2D6, an important enzyme for approximately $25 \%$ of all drugs administered in clinical practice. The aim of this review is to summarize data on the impact of CYP2D6 polymorphism on pharmacokinetics, pharmacodynamics and adverse effects of weak opioids.
\end{abstract}

Copyright $\odot 2011$ S. Karger AG, Basel

\section{Introduction}

Cytochrome P450 (CYP) consists of hem-containing monooxygenase isoenzymes located on the smooth endoplasmic reticulum membranes of liver hepatocytes and along the mucosal surface of the intestinal tract [1]. These enzymes take part in the endogenous metabolism of steroids, hormones, prostaglandins and lipids and in the detoxification of exogenous compounds. The CYP system is responsible for type I reactions which can inactivate or activate a given drug [2]. The type II systems comprise conjugates (glucuronidation and sulfation) connected with drug excretion. Along with CYP3A4, the most important enzyme involved in the metabolism of over $50 \%$ of all drugs, CYP2D6 is vital as well for the metabolism of clinically used drugs [3]. Although it accounts only for $2-5 \%$ of the total hepatic P450 isoenzymes, it also accounts for $25 \%$ of metabolized drugs [4]. It is also important for many drugs used in pain and palliative medicine as it is responsible for metabolizing certain opioids, neuroleptics, antidepressants and cardiac medications [5]. CYP2D6-metabolized drugs exhibit nonlinear saturable kinetics owing to the low capacity of CYP2D6 [6]. The enzyme is highly polymorphic.

\section{KARGER}

Fax +41613061234 E-Mail karger@karger.ch www.karger.com
Wojciech Leppert

Chair and Department of Palliative Medicine

Poznan University of Medical Sciences

Osiedle Rusa 25 A, PL-61 245 Poznan (Poland)

Tel. +48 618738 303, E-Mail wojciechleppert@wp.pl 
More than 80 distinct allelic variants for CYP2D6 are known, which leads to a wide spectrum of metabolic capacity and phenotype diversity within populations $[7,8]$. Individuals carrying two wild-type alleles display normal enzyme activity and are known as extensive metabolizers (EMs). Poor metabolizers (PMs) display two inactive alleles and are characterized by deficient hydroxylation of several classes of drugs, such as $\beta$-blockers, antiarrhythmics, antidepressants, neuroleptics and some opioid analgesics. In approximately $7-10 \%$ of the Caucasian populations, an autosomal recessive trait of nonfunctional alleles is present $[9,10]$. These PMs are at an increased risk of sustaining excessive pharmacodynamic and adverse effects due to a relative drug overdose when the parent compound is responsible for the therapeutic effects [11]. When the prodrug is metabolized to its active metabolite(s) therapeutic failure may be observed in PMs [12]. In comparison to Caucasians, the incidence of PMs is much lower in Asian and African populations. Caucasians have a significantly increased frequency of three defective genes: CYP2D6*4, CYP2D6*3 and CYP2D6*6 whereas the frequency of CYP2D $6 * 5$ defective alleles is similar to that of other ethnic groups, all contributing to the PM phenotype [13].

The duplication or multiduplication of the CYPD6 gene (mostly CYP2D6* 1 and CYP2D6* 2 alleles in Caucasians) is associated with an ultrarapid metabolism of some compounds. Ultrarapid metabolizers (UMs) may experience either a lack of efficacy if the parent compound is responsible for the therapeutic effect of a given drug or very intense therapeutic effects associated with the production of an excessive amount of active metabolite(s) that may also be responsible for intense adverse effects. The incidence of UMs is low in northern (1-2\%), middle Europe, North America (4-5\%) and Asia $(0.5-2.5 \%)$ but is significantly higher in Mediterranean (7-12\%), Saudi-Arabian (21\%) and Ethiopian (29\%) populations [14]. Other polymorphisms include CYP2D6*10 that leads to instable enzyme activity with a high occurrence (41-51\%) in Asian populations and CYP2D6*17 that leads to reduced affinity for substrates with a high incidence in African populations (20-35\%); both are responsible for the intermediate metabolizer (IM) phenotype $[15,16]$. The IM phenotype may also be relevant to the clinical effects of CYP2D6 substrates although to a lesser extent when compared with PM and UM phenotypes.

\section{Tramadol}

Tramadol (1RS,2RS)-2-[(dimethylamino)methyl]-1-(3methoxyphenyl)-cyclo-hexanol is a synthetic opioid of the aminocyclohexanol group, an analgesic with opioid agonist properties and acting on noradrenalin and serotonin neurotransmission $[17,18]$. Tramadol is a racemic mixture; (-)-tramadol is about 10 times more potent than $(+)$-tramadol in inhibiting noradrenalin uptake and (+)-tramadol is about 4 times stronger than (-)-tramadol in inhibiting serotonin uptake. Both enantiomers act synergistically to improve analgesia without increasing the adverse effects [19].

Tramadol is mainly metabolized by the CYP enzyme system in the liver and excreted by the kidneys. Tramadol undergoes biotransformation in the liver, firstly by the phase I reactions (mainly $\mathrm{O}$ - and $\mathrm{N}$-demethylation), and secondly by the phase II reactions (mainly conjugation of $\mathrm{O}$ - and $\mathrm{N}$-demethylated compounds) [20,21]. In the phase I reactions, 11 metabolites and in the phase II reactions, 12 metabolites are produced; the main metabolite is $\mathrm{O}$ desmethyltramadol $\left(\mathrm{M}_{1}\right)$ [22]. It shows analgesic activity and has a higher affinity for $\mu$-opioid receptors than the parent compound $[23,24] ;(+)-\mathrm{M}_{1}$ has $300-400$ times greater affinity for $\mu$-opioid receptors than tramadol [25] whereas (-)- $\mathrm{M}_{1}$ mainly inhibits noradrenalin reuptake [26]. Apart from $\mathrm{O}, \mathrm{N}$-didesmethyltramadol $\left(\mathrm{M}_{5}\right.$, which exhibits weaker analgesic activity than $\mathrm{M}_{1}$ ), other metabolites are pharmacologically inactive. Mono-O-demethylation leading to $\mathrm{M}_{1}$ production is possible owing to the polymorphic CYP2D6 enzyme (sparteine oxygenase) of cytochrome $\mathrm{P} 450$ in the liver, which is inhibited by quinidine, a selective inhibitor of this enzyme [27]. The elimination half-life of tramadol is about 5-6 h and that of $\mathrm{M}_{1}$

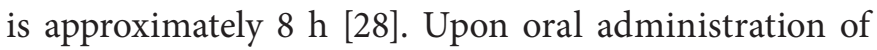
tramadol, about $90 \%$ of the drug is excreted by the kidneys and $10 \%$ with the feces [21]. Patients with renal impairment (creatinine clearance $<79 \mathrm{ml} / \mathrm{min}$ ) show a decreased excretion of tramadol and $M_{1}$ in comparison to healthy individuals with normal renal function (creatinine clearance $>100 \mathrm{ml} / \mathrm{min}$ ) [27]. In patients with advanced cirrhosis, there is a decrease in tramadol metabolism with a concomitant decrease in hepatic clearance and a rise in the blood serum levels. In these patients a 2.5 -fold increase in the elimination half-life is observed [29]. The starting dose of immediate-release (IR) tramadol is about 25-50 mg every $4-6 \mathrm{~h}$ and, in the case of controlled-release (CR) tablets or capsules, 50-100 mg twice daily [28].

Studies conducted in patients with postoperative pain demonstrated that patients devoid of CYP2D6 activity 


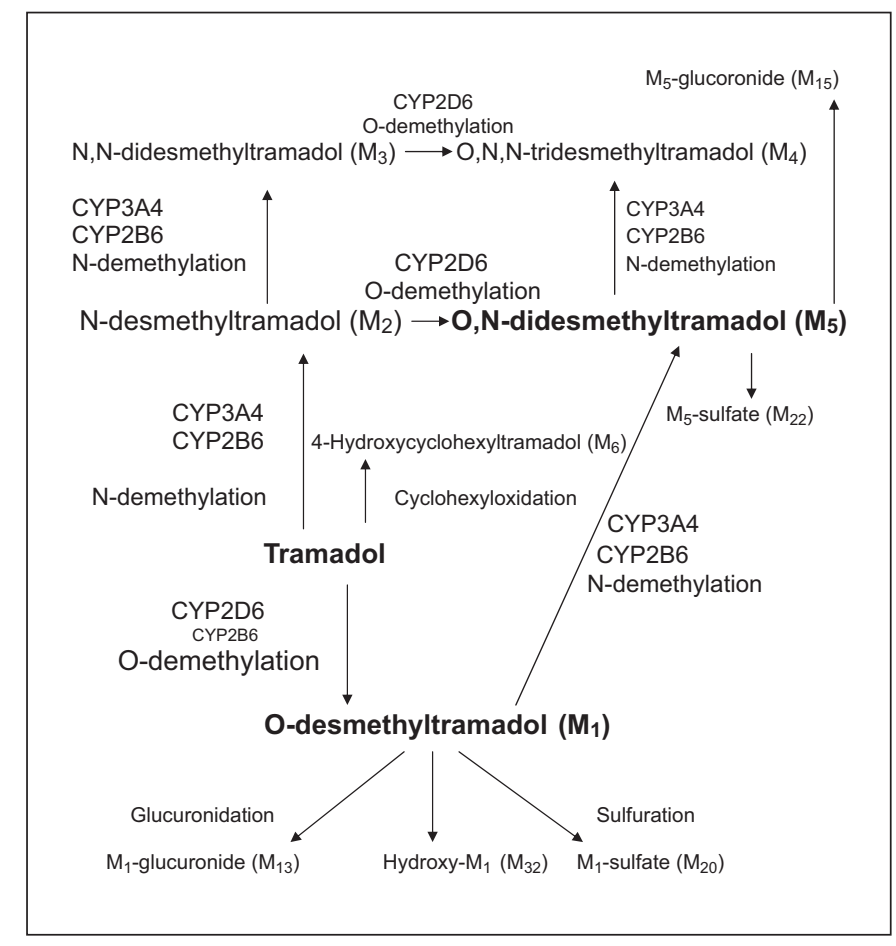

Fig. 1. Main metabolic pathways of tramadol, adapted from Subrahmanyam et al. [24] and Klotz et al. [28]. The main compounds responsible for tramadol analgesia are marked in bold.

(PMs) need approximately 30\% higher tramadol doses than those with normal CYP2D6 activity (EMs) [30]. Assays of tramadol and $\mathrm{M}_{1}$ enantiomers conducted in healthy volunteers and in patients with postoperative pain [30] demonstrated that tramadol analgesia depends on CYP2D6 genotype, with less analgesic effects observed in PM, which is associated with a lack of $(+)-\mathrm{M}_{1}$ enantiomer formation that is responsible for the opioid component of tramadol analgesia [31]. Genotyping is helpful in patients with duplication of the CYP2D6 gene (UMs) as these patients are at greater risk to develop adverse effects to tramadol $[14,32]$. Tramadol is also metabolized through CYP3A4 and CYP2B6 to N-desmethyltramadol $\left(\mathrm{M}_{2}\right)$, and through CYP2D6 and CYP3A4 to $\mathrm{M}_{5}$ (fig. 1) [27, 33, 34].

Stamer et al. [30] investigated whether the PM genotype had an impact on the response to tramadol in 300 postoperative patients treated with a $1-\mathrm{ml}$ bolus dose of a combination of tramadol $20 \mathrm{mg} / \mathrm{ml}$, dypirone $200 \mathrm{mg} / \mathrm{ml}$ and metoclopramide $0.4 \mathrm{mg} / \mathrm{ml}$ via patient-controlled analgesia after titration to an individual loading dose. $\mathrm{Pa}$ tients classified as PMs $(n=30)$ needed higher loading doses of tramadol than patients classified as EMs ( $\mathrm{n}=$
$241 ; 144.7 \pm 22.6$ and $108.2 \pm 56.9 \mathrm{mg}$, respectively; $\mathrm{p}<$ $0.001)$; the percentage of nonresponders was significantly higher in the PM group (46.7 vs. $21.6 \%$; $\mathrm{p}<0.005$ ); more patients from the PM group needed rescue analgesia in the recovery room $(43.3$ vs. $21.6 \%$; $p<0.02)$. In another study, tramadol was administered intravenously at a dose of $3 \mathrm{mg} / \mathrm{kg}$ for postoperative analgesia in 170 patients. The concentration of $\mathrm{M}_{1}$ differed between PMs, IMs, EMs and UMs. Median (1/3 quartile) area under the concentration-time curves for (+)- $\mathrm{M}_{1}$ were $0(0 / 11.4), 38.6$ (15.9/75.3), 66.5 (17.1/118.4), and $149.7(35.4 / 235.4) \mathrm{ng} \times$ $\mathrm{h} / \mathrm{ml}$ for PMs, IMs, EMs and UMs, respectively $(\mathrm{p}<$ 0.001). Medications inhibiting CYP2D6 administered with tramadol decreased $(+)-\mathrm{M}_{1}$ concentrations $(\mathrm{p}<$ 0.01). In PMs nonresponder rates to tramadol treatment increased fourfold compared with the other genotypes $(\mathrm{p}<0.001)$ [31].

Wang et al. [15] investigated whether the CYP2D6*10 allele had an impact on the postoperative analgesia effect of tramadol in 70 Chinese patients after gastrectomy. The allele frequency of CYP2D6*10 is $52.4 \%$; patients were categorized into three groups according to the CYP2D6 genotype: patients without CYP2D6*10 (group 1; $\mathrm{n}=17$ ), patients heterozygous for CYP2D6*10 (group $2 ; \mathrm{n}=26$ ), and patients homozygous for CYP2D6*10 (group 3; $\mathrm{n}=$ 20). The demographic data of the three groups were comparable. The total consumption of tramadol for $48 \mathrm{~h}$ in group 3 was significantly higher than that in groups 1 and 2 , while it did not differ between groups 1 and 2 . The CYP2D6*10 allele has a significant impact on analgesia with tramadol in a Chinese population and pharmacogenetics may explain some of the varying responses to analgesics in patients with postoperative pain.

Gan et al. [16] investigated the influence of the CYP2D6*10 allele on the disposition of tramadol hydrochloride in Malaysian subjects. A single dose of $100 \mathrm{mg}$ tramadol was given intravenously to 30 healthy orthopedic patients undergoing various elective surgeries. Patients were genotyped for CYP2D6*10 and the presence of CYP2D6 ${ }^{*} 1,{ }^{*} 3,{ }^{*} 4,{ }^{*} 5,{ }^{*} 9$ and ${ }^{*} 17$ mutations. The pharmacokinetics of tramadol was studied during $24 \mathrm{~h}$ after drug administration. The allele frequency for CYP2D6*10 was high (0.43). Subjects who were homozygous for CYP2D6*10 had significantly $(p=0.046)$ longer mean serum half-lives of tramadol $(12.1 \mathrm{~h})$ than subjects of the normal $(7.2 \mathrm{~h})$ or heterozygous group $(10 \mathrm{~h})$. When patients were screened for the presence of other alleles, the values of the pharmacokinetic parameters were more easily explained. The CYP2D6*10 allele, in particular, was associated with high serum levels of 
tramadol compared with the CYP2D6* 1 allele but genotyping for CYP2D6*10 alone is not sufficient to explain tramadol disposition.

Tramadol metabolism through the CYP2D6 enzyme of CYP in the liver can be a reason for possible interactions with drugs that inhibit this enzyme $[33,34]$. This applies to two commonly used drugs, i.e. cimetidine and ranitidine. Combination of tramadol with selective serotonin reuptake inhibitors (SSRIs; fluoxetine, paroxetine and to a lesser extent sertraline) inhibits CYP2D6 and may cause the serotonin syndrome because SSRIs, apart from inhibiting tramadol metabolism, increase the level of serotonin in the CNS; they should not be coadministered with tramadol. The serotonin syndrome may also appear with concomitant administration of monoamine oxidase inhibitors, olanzapine, risperidone and venlafaxine [35-37]. On the other hand, mianserin and mirtazapine do not influence serotonin level and do not inhibit CYP2D6, but they are substrates of this enzyme [38].

Inhibition of tramadol metabolism may attenuate analgesia because (+)- $\mathrm{M}_{1}$ has significant opioid analgesic activity. Attenuation of tramadol analgesia can be caused by concomitant administration of ondansetron (a selective antagonist of the type 3 serotonin, $5 \mathrm{HT}_{3}$, receptor) as it blocks spinal $5 \mathrm{HT}_{3}$ receptors and competitively inhibits CYP2D6 [39]. Tramadol analgesia is impaired by concomitant administration of carbamazepine due to the acceleration of tramadol and $\mathrm{M}_{1}$ metabolism [40]. Concomitant administration of tricyclic antidepressants increases the risk of seizures. Tramadol should be avoided in patients with a history of epilepsy. However, tramadol administered alone does not influence the possibility of fits [41]. In rats and mice, concomitant administration of tramadol and pindolol, a $\beta$-blocker and 5HT1A/1B antagonist, enhances analgesia [42]. According to Sindrup et al. [43], the opioid effect of (+)- $M_{1}$ may be of importance for tramadol-induced relief of ongoing neuropathic pain but, in general, relief of neuropathic pain seems to depend on both the monoaminergic effect of $(+)$ - and $(-)$-tramadol and the opioid effect of $(+)-\mathrm{M}_{1}[43]$.

Poulsen et al. [44] assessed the analgesic effect of $2 \mathrm{mg} /$ $\mathrm{kg}$ tramadol in $15 \mathrm{EMs}$ and $12 \mathrm{PMs}$ in two parallel, randomized, double-blind, placebo-controlled crossover studies that used experimental pain models. In EMs, tramadol increased pressure pain detection $(\mathrm{p}=0.03)$ and tolerance $(\mathrm{p}=0.06)$ thresholds, as well as thresholds for eliciting nociceptive reflexes, after single $(\mathrm{p}=0.0002)$ and repeated $(p=0.06)$ stimulation of the sural nerve. Peak pain and peak area in the cold pressor test were reduced
( $p=0.0006$ and $p=0.0009)$. In PMs, only pressure pain tolerance thresholds $(\mathrm{p}=0.02)$ and nociceptive reflexes were increased after single stimulation $(\mathrm{p}=0.04)$ and reflex thresholds were increased in PMs to a lesser extent than in EMs $(p=0.02)$. Serum concentrations of $(+)-M_{1}$ within $2-10 \mathrm{~h}$ after tramadol administration ranged from 10 to $100 \mathrm{ng} / \mathrm{l}$ in EMs whereas in PMs serum concentrations of $(+)-\mathrm{M}_{1}$ were below or around the detection limit of $3 \mathrm{ng} / \mathrm{ml}$. Formation of $(+)-\mathrm{M}_{1}$ via CYP2D6 is important for the effect of tramadol in experimental pain.

Kirchheiner et al. [14] tested the impact of the CYP2D6 gene duplication on tramadol pharmacokinetics and pharmacodynamics. A single tramadol dose of $100 \mathrm{mg}$ was administered to 11 carriers of a CYP2D6 gene duplication allele (UMs) and compared with 11 carriers of the two active CYP2D6 genes (EMs). The maximum plasma concentrations of $(+)-\mathrm{M}_{1}$ were significantly higher in the UM group compared with the EM group $(\mathrm{p}<$ 0.005 ; t test) with a mean difference of $14 \mathrm{ng} / \mathrm{ml}(95 \% \mathrm{CI}$, 2-26 ng/ml). The median (+)-tramadol area under the curve (AUC) was 786 and $587 \mu \mathrm{g} \cdot \mathrm{h} \cdot \mathrm{liter}^{-1}$ in EM and UM, and the corresponding median (+)- $\mathrm{M}_{1}$ AUC was 416 and $448 \mu \mathrm{g} \cdot \mathrm{h} \cdot \mathrm{liter}^{-1}$ ( $\mathrm{p}<0.005$; $\mathrm{t}$ test). There was an increased pain threshold and pain tolerance and a stronger miosis after tramadol in UMs compared with EMs. Almost 50\% of the UM group experienced nausea compared with $9 \%$ of the EM group. Pharmacokinetic differences between EMs and UMs were smaller than expected; nevertheless, UMs were more sensitive to tramadol than EMs. Tramadol frequently causes adverse effects in southern European and northern African populations with a high proportion of UMs.

Respiratory depression is a rare occurrence in the chronic use of tramadol; however, high tramadol doses used in cats $(4 \mathrm{mg} / \mathrm{kg}$ ) suggest this possibility [45]. Respiratory depression was observed during tramadol treatment in patients with cancer pain and renal impairment [46]. This is associated with the accumulation of the active metabolite $\left(M_{1}\right)$, which has a longer elimination halflife than the parent compound and binds to $\mu$-opioid receptors. Respiratory depression is connected with the opioid mode of tramadol action so if it does occur, naloxone should be administered intravenously. Respiratory depression in a patient treated with tramadol for postoperative pain due to resection of renal carcinoma, with renal impairment (creatinine clearance $30 \mathrm{ml} / \mathrm{min}$ ) and with UM genotype was depicted. As the symptom appeared after more than $10 \mathrm{~h}$ following the first tramadol dose, the accumulation of $\mathrm{M}_{1}$ was claimed to be the cause; the patient recovered after administration of an intrave- 


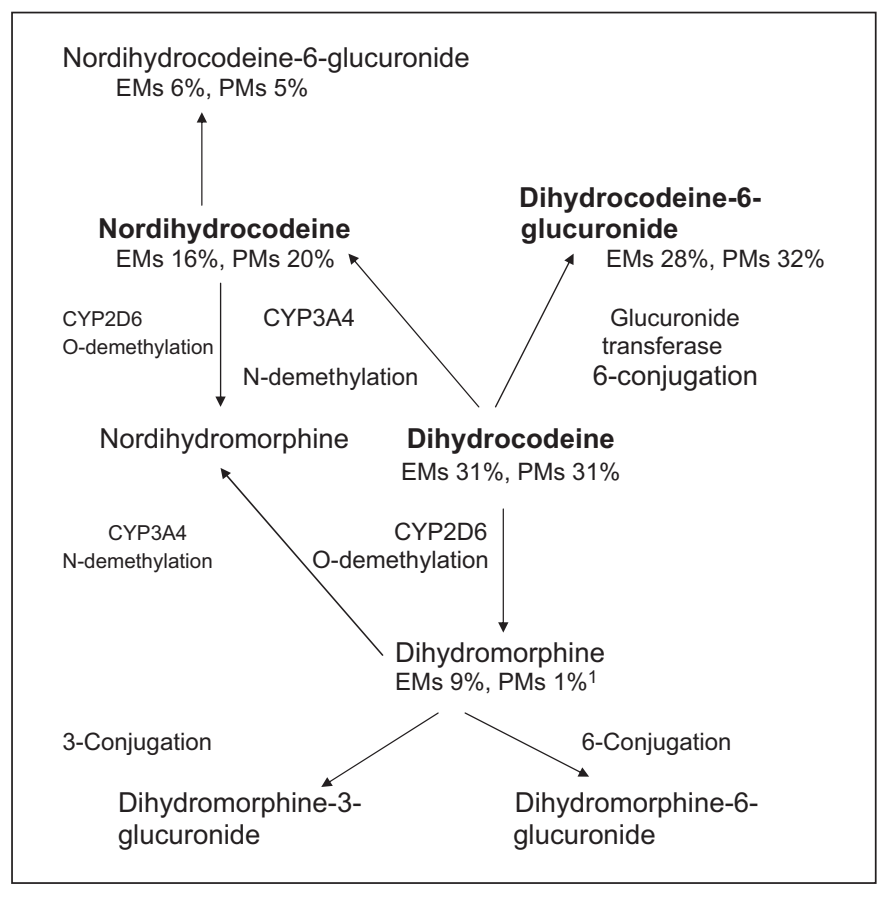

Fig. 2. DHC metabolism, modified. The most important compounds responsible for DHC analgesia are marked in bold. ${ }^{1}$ This percentage refers to DHM and its metabolites. Percentages of a single oral DHC dose found in the urine in EMs and PMs are shown along with the compounds.

nous naloxone bolus $(0.4 \mathrm{mg})$. Authors do not recommend tramadol administration in patients with UM genotype and renal impairment [32]. These data indicate that tramadol analgesia is dependent on CYP2D6 activity [47-49].

\section{Dihydrocodeine}

Dihydrocodeine (DHC) is a semi-synthetic analogue of codeine. In patients with postoperative pain, analgesia after subcutaneous administration of $30 \mathrm{mg}$ DHC is similar to analgesia induced by $10 \mathrm{mg}$ of morphine [50]. Apart from its analgesic [51] and antitussive activity, DHC is also used in the treatment of opioid addiction [52]. After parenteral administration, DHC is twice as potent as codeine. The bioavailability of DHC after oral administration is about $20 \%$, which indicates that analgesia after oral administration is slightly stronger than that induced by codeine (its bioavailability after oral administration equals approximately 30-40\%). After oral administration of DHC, the maximal serum concentration appears after
$1.7 \mathrm{~h}$, plasma half-life varies from 3.5 to $5.5 \mathrm{~h}$, analgesia lasts $4 \mathrm{~h}$. Ammon et al. [53] assessed DHC pharmacokinetics in 12 healthy volunteers who were EMs of CYP2D6. They received a single DHC dose of $60 \mathrm{mg}$, then after $60 \mathrm{~h}$, they were administered a $60-\mathrm{mg}$ dose twice daily for 3 days, for the next 3 days a dose of $90 \mathrm{mg}$ twice daily and for 3 days a dose of $120 \mathrm{mg}$ twice daily (CR 60-, 90- and 120 -mg tablets) orally. In the examined DHC dose range $(60-120 \mathrm{mg})$, the pharmacokinetics of DHC and dihydromorphine (DHM) displayed linear characteristics: AUC, $\mathrm{C}_{\max }$ and $\mathrm{C}_{\text {ssmin }}$ for both compounds increased depending on the drug dose [54]. Even though DHM displays higher affinity (about 100-fold) for $\mu$-opioid receptors and exhibits higher analgesic activity than the parent compound, the role of DHM and its glucuronides in DHC analgesia has not been unequivocally established yet. The starting dose of IR DHC is $30 \mathrm{mg}$ every $4-6 \mathrm{~h}$ and that of the CR DHC is one 60-mg tablet twice daily.

Renal clearance and clearance of DHC metabolites: glucuronidation and O-demethylation to dihydrocodeine-6-glucuronide (DHC-6-G) and DHM, respectively, are not dose dependent, which indicates that the metabolism and excretion of DHC and its metabolites is not dose dependent. The ratio of DHC to DHM for AUC did not change depending on the dose, which suggests the lack of a saturation effect of the O-demethylation of DHC to DHM depending on CYP2D6 in EMs. Pharmacokinetic parameters were similar after a single dose and multiple doses of $60 \mathrm{mg}$ of DHC [53]. Frazer et al. [55] in a randomized cross-over study in healthy volunteers compared the single-dose and a multiple-dose pharmacokinetics of IR and CR DHC formulations. The results confirmed the CR characteristics of DHC Continus and provide evidence in favor of a twice-daily dosage schedule. DHC is metabolized in the liver to three main metabolites: DHM, DHC-6-G and nordihydrocodeine (NORDHC). NORDHC is further glucuronidated to NORDHC-6-glucuronide and O-demethylated to nordihydromorphine. DHM undergoes glucuronidation to dihydromorphine-3-glucuronide (DHM-3-G), and dihydromorphine-6-glucuronide (DHM-6-G) and N-demethylation to nordihydromorphine. It may be concluded that DHC undergoes the first-pass effect after oral administration, which is connected with the formation of a significantly higher amount of metabolites after oral than after parenteral administration [54]. DHC metabolism is schematized in figure $2[56,57]$.

Schmidt et al. [58] evaluated the affinity of DHC and its metabolites: DHC-6-G, DHM, DHM-3-G, DHM$6-\mathrm{G}, \mathrm{NORDHC}$ for $\mu-, \delta$ - and $\kappa$-opioid receptors in the 
brain of the guinea pig. All substances displayed the greatest affinity for $\mu$-opioid receptors, and, with the exception of morphine, the least affinity for $\mathrm{\kappa}$-opioid receptors. Of the explored compounds, the highest affinity for $\mu$-opioid receptors was displayed by DHM, DHM6-G and morphine. The affinity of DHM and DHM-6-G for $\mu$-opioid receptors was at least 70 times more potent whereas other metabolites displayed less affinity as compared to DHC. DHM-6-G had a little lower whereas DHM-3-G significantly lower affinity for $\mu$-opioid receptors than DHM. DHC and DHM displayed affinity that was twice as high as that of their 7,8-nonsaturated analogues (codeine and morphine). In conclusion, the O-demethylation of DHCleads to the formation of DHM and DHM-6-G, which displays the highest affinity for $\mu$-opioid receptors. This reaction depends on CYP2D6 enzyme activity.

In a randomized, cross-over, placebo-controlled study, Webb et al. [59] assessed the influence of DHC and DHM on analgesia using a cold-induced pain test in 10 healthy volunteers after administration of a single oral 90-mg DHC dose. They found that DHC contributed significantly more to the analgesic effect due to its over 50 times higher serum concentration compared to DHM. Moreover, one of the volunteers did not metabolize the drug at all (PM) but analgesia observed in this patient was similar to that in all other volunteers. The indirect evidence was also the fact that the large interindividual variation (up to 20-fold) in DHM plasma concentrations was not related to the large interindividual variation (7-fold) in analgesia following DHC administration.

Jurna et al. [60] assessed the inhibition of pain evoked by electrical stimulation of afferent $C$ fibers of the sural nerve by measuring electrical impulses of neurons in the thalamus nuclei in rats. Analgesia was caused by the intravenous administration of DHC $(0.25,0.5,1$ and $2 \mathrm{mg} /$ $\mathrm{kg}), \operatorname{DHM}(0.5,1,2$ and $4 \mathrm{mg} / \mathrm{kg})$ and morphine $(0.5 \mathrm{mg} /$ $\mathrm{kg}$ ), alone or with pretreatment with drugs inhibiting $\mathrm{O}$ demethylation of DHC: metyrapone and cimetidine. Intrathecal administration of DHC and DHM at a dose of $0.1 \mathrm{mg}$ was compared. Intravenous administration of DHC $2 \mathrm{mg} / \mathrm{kg}$, DHM $4 \mathrm{mg} / \mathrm{kg}$ and morphine alone completely abolished the activity of thalamic neurons in a dose-dependent manner. DHC displayed 2-fold stronger analgesia than DHM (ED $\mathrm{ED}_{50}$ for DHC $0.47 \mathrm{mg} / \mathrm{kg}$ vs. 0.97 $\mathrm{mg} / \mathrm{kg}$ for DHM). Metyrapone and cimetidine did not influence neuronal activity; only cimetidine, administered before DHC injection $(2 \mathrm{mg} / \mathrm{kg})$ at the beginning of the observation, attenuated the analgesia. Naloxone, at a dose of $0.2 \mathrm{mg} / \mathrm{kg}$, reversed the effect of DHC. Intra- thecal administration of DHC and DHM caused a similar analgesic effect although analgesia after DHM administration lasted longer. DHC produces analgesia by itself; this effect is due to its binding to opioid receptors as it is reversed by naloxone. Basing on the $\mathrm{ED}_{50}$ value and the average body mass of an adult human $(70 \mathrm{~kg})$, it was proposed that a single DHC dose should be at least $30 \mathrm{mg}$.

Wilder-Smith et al. [61] established the contribution of the DHM metabolite to DHC analgesia by investigating the effects of DHC on somatic and visceral pain thresholds in 11 healthy volunteers (EMs and PMs) in a doubleblind, randomized, placebo-controlled, four-way crossover study. The authors compared the effects of single doses of placebo and $60 \mathrm{mg}$ CR DHC with and without premedication with quinidine sulfate $50 \mathrm{mg}$ on electrical, heat and rectal distension pain tolerance thresholds. DHC significantly raised the heat pain tolerance thresholds (at 3.3 and $5 \mathrm{~h}$ after dosing; $\mathrm{p}<0.05$ ), rectal distension defecatory urge (at 3.3 and $10 \mathrm{~h}$ after dosing; $\mathrm{p}<0.02$ ) and pain tolerance thresholds (at 3.3 and $5 \mathrm{~h}$ after dosing; $\mathrm{p}<0.05)$ as compared with placebo. Premedication with quinidine did not change the effects of $\mathrm{DHC}$ on pain thresholds, but decreased the effect of DHC on defecatory urge thresholds (at 1.5, 3.3 and $10 \mathrm{~h}$ after dosing; $\mathrm{p}<$ 0.05). Similar levels of DHC in EMs and in PMs were observed. However, levels of DHM and its metabolites were 3-4 times higher in EMs as compared to PMs. The amount of DHM in EM urine was $0.91 \%$ of the DHC dose versus $0.28 \%$ in PM urine. DHM does not play a significant role in DHC analgesia.

Kirkwood et al. [62] assessed the kinetics of DHM and NORDHC formation and the role of individual P450 cytochrome enzymes in the liver microsomes in EMs and PMs (in vitro study). N-demethylation to NORDHC was the predominant metabolic pathway of $\mathrm{DHC}$ in all patients (both EMs and PMs). The reaction of O-demethylation was much slower than $\mathrm{N}$-demethylation in both PMs and EMs. Quinidine and quinine were potent inhibitors of O-demethylation in EMs but not in PMs. None of the remaining compounds inhibited DHM formation in either EMs or PMs. DHM formation might be explained by the two-enzyme model because in PMs DHM was also present in a small but detectable amount. The main oxidative metabolic pathways of DHC are N-demethylation to NORDHC catalyzed by CYP3A4 and Odemethylation to DHM catalyzed predominantly by CYP2D6 with a small participation of another enzyme. DHM formation depends on CYP2D6 polymorphism and DHM is approximately 100 times more potent than 
DHC and its other metabolites (NORDHC and DHC6-G) and displays similar potency to morphine.

Kirkwood et al. [63] assessed the usefulness of liquid chromatography for assays of DHC-6-G, characteristics of DHC-6-G formation kinetics and the inhibitory effect of some drugs that may be coadministered with DHC and that are substrates of glucuronide transferase in liver microsomes taken from patients after liver resection due to cancer. The $\mathrm{K}_{\mathrm{i}}$ values (dissociation constant) were similar to the respective values for codeine. The strongest inhibitory effect on DHC-6-G formation was displayed by diclofenac, followed by amitryptiline, oxazepam and naproxen; paracetamol did not influence DHC-6-G formation.

Schmidt et al. [56], in a randomized, double-blind, cross-over and two-part (drug and placebo) clinical trial assessed the pharmacokinetics of DHC and metabolites (DHC-6-G, DHM, DHM-3-G, DHM-6-G and NORDHC) and analgesia in young, healthy volunteers (5 EMs and 4 PMs) after a single 60-mg oral dose of DHC. The affinities of DHM and DHM-6-G were approximately 100 -fold and 50-fold higher, respectively, than that of DHC, whereas the other metabolites had lower affinities as compared to DHC. DHM showed 3 -fold greater activity than its 7,8-unsaturated analogue, morphine, whereas DHC, DHC-6-G and the 7,8-unsaturated analogue, codeine, had similar potencies. No differences were found between EMs and PMs concerning AUC, $\mathrm{C}_{\max }, \mathrm{T}_{\max }$ and serum half-life of DHC and DHC-6-G. The main DHC metabolite was DHC-6-G in both EMs and PMs. DHM was only found in the plasma in EMs ( $\mathrm{T}_{\max } 1.5 \mathrm{~h}$ ); however, after $6 \mathrm{~h}, \mathrm{DHM}$ was no longer detectable in the plasma of EMs. DHM was further metabolized to DHM-3-G $\left(\mathrm{T}_{\max } 2 \mathrm{~h}\right)$ and DHC-3-G was still measurable after $12 \mathrm{~h}$ (serum half-life 5.8 h). DHM, DHM-3-G or DHM-6-G was not found in PMs. In the urine, the major DHC metabolite was DHC-6-G (more than $50 \%$ of the DHC dose was excreted as DHC-6-G within $24 \mathrm{~h}$ ), $20-24 \%$ of the dose was excreted as DHC (no difference between EMs and PMs). Maximal renal excretion of DHC was found from 0 to $2 \mathrm{~h}$ whereas the excretion of metabolites was maximal between 4 and $8 \mathrm{~h}$. Significant differences were observed concerning the urinary excretion of NORDHC in EMs $(11.8 \pm 2.3 \mu \mathrm{mol} / 24 \mathrm{~h})$ versus PMs $(22.0 \pm 2.8$ $\mu \mathrm{mol} / 24 \mathrm{~h} ; \mathrm{p}<0.05)$. DHM and its metabolites were not found in the urine of PMs. The pupil diameter was similarly reduced in EMs and PMs after DHC administration up to $6 \mathrm{~h}$ versus placebo $(\mathrm{p}<0.05)$. In the pain threshold model and subjective rating of pain intensity, no differences between placebo and $60 \mathrm{mg}$ of $\mathrm{DHC}$ and between
EMs and PMs were found. The O-demethylated DHC metabolites display greater affinity for opioid receptors and pharmacological activity in comparison to the parent compound, and CYP2D6 is crucial for DHM formation. In spite of their high pharmacological activity, DHM and DHM-6-G do not make a major contribution to the analgesic effects of DHC. Studies performed to date [56, 59-61] indicate that DHC analgesia is independent of CYP2D6 activity [64].

\section{Codeine}

Codeine is a methylated morphine derivative that is found naturally along with morphine in the poppy seed [64]. Codeine displays analgesic and antitussive activity. Codeine is available as IR and CR formulations but also as a combination with paracetamol (effervescent tablets combine $30 \mathrm{mg}$ of codeine and $500 \mathrm{mg}$ of paracetamol). The addition of codeine to paracetamol enhances paracetamol analgesia [65]. IR codeine is administered every $4-6 \mathrm{~h}$ in chronic pain with a starting single dose of 20-30 mg. The daily doses of DHC and codeine usually do not exceed 240 and $300 \mathrm{mg}$, respectively; when these analgesics are ineffective, opioids for moderate to severe pain (strong opioids) are introduced.

Codeine displays approximately $30-40 \%$ bioavailability after oral administration and is metabolized in the liver. Following oral administration, the maximal plasma concentration is attained within $1-2 \mathrm{~h}$ with a plasma half-life of 2.5-3.5 $\mathrm{h}$ and analgesia maintained for approximately $4-6 \mathrm{~h}$ (IR formulations). Codeine is partially metabolized to morphine and its metabolites and to codeine metabolites: norcodeine (NORC) and codeine-6-glucuronide(C-6-G) [66]. The analgesic effect of codeine equals approximately $1 / 10$ th of morphine analgesia. The polymorphism of CYP2D6 that is responsible for morphine and the formation of its metabolites may affect codeine analgesia. However, other codeine metabolites, predominantly C-6-G, also display significant analgesic activity and contribute to codeine analgesia [67]. In healthy volunteers, codeine is metabolized to C-6-G $(81.0 \pm 9.3 \%)$, NORC (2.16 $\pm 1.44 \%)$, morphine $(0.50 \pm 0.39 \%)$, morphine-3-glucuronide (M3-G) (2.10 $\pm 1.24 \%)$, morphine-6-glucuronide (M-6-G) $(0.80 \pm 0.63 \%)$ and normorphine $(2.44 \pm 2.42 \%)$. The half-life of codeine is $1.47 \pm 0.32 \mathrm{~h}$, that of C-6-G 2.75 $\pm 0.79 \mathrm{~h}$. The plasma AUC of C-6-G is approximately 10 -fold higher than that of codeine. Protein binding of codeine and C-6-G in vivo was $56.1 \pm 2.5$ and $34.0 \pm$ 
$3.6 \%$, respectively [68]. Codeine metabolic pathways are shown in figure 3.

Lötsch et al. [66] explored the contribution of codeine and its metabolites to central nervous analgesic effects independent of $\mathrm{O}$-demethylation of codeine to morphine. Eleven healthy volunteers representing PMs, IMs, EMs and UMs received oral codeine solution at a dose of 80 $\mathrm{mg}$ increased to $120 \mathrm{mg}$ due to insufficient miotic effects. The observed plasma morphine concentrations were mimicked by the use of a computerized morphine infusion, and the miotic effects were compared with those observed after codeine administration. The contribution of codeine, C-6-G, NORC, morphine, M-6-G and normorphine to the miotic effects was analyzed by means of pharmacokinetic-pharmacodynamic (PK-PD) modeling. The AUCs of the miotic effect after codeine administration were $1.7 \pm 2$ times greater than those after morphine $(\mathrm{p}<0.01)$. This contrasted to similar or lower morphine concentrations after codeine than after morphine (the AUC ratio, $0.5 \pm 0.4 ; \mathrm{p}=0.21$ ). A PK/PD fit of the miotic effects of morphine as the only active compound was most significantly $(\mathrm{p}<0.0001)$ improved when $\mathrm{C}-6-\mathrm{G}$ was added as the second active moiety. CYP2D6-dependent formation of morphine does not explain exclusively the central nervous effects of codeine, and C-6-G is the most likely additional active moiety with a possible contribution of NORC and the parent compound.

Gasche et al. [69] reported a patient who received oral codeine at a daily dose of $75 \mathrm{mg}$ ( $25 \mathrm{mg}$ three times a day) and who experienced symptoms of drug overdose (lack of consciousness, respiratory depression) after 4 days of treatment. The patient recovered after intravenous administration of naloxone $(0.4 \mathrm{mg})$. The cause of these symptoms was his CYP2D6 UM phenotype as genotyping showed 3 or more functional alleles. The patient was concomitantly treated with a macrolide (clarithromycin) and an azole derivative (voriconazole), both known inhibitors of CYP3A4 as confirmed by low CYP3A4 activity. This, together with CYP2D6 gene duplication, led to the reduced clearance of codeine. Blood concentrations of morphine metabolites: $M-3-G$ and $M-6-G$ were substantially elevated, a finding also likely to be attributable to renal failure. The total amount of morphine and metabolites corresponded to $75 \%$ of the total amount of codeine present in the patient's body whereas the usual amount of morphine that is produced after the administration of multiple doses of codeine in a person with EM CYP2D6 reaches $10 \%$.

Kirchheiner et al. [70] conducted a study in volunteers (12 UMs, 11 EMs, and 3 PMs of CYP2D6) who were ad-

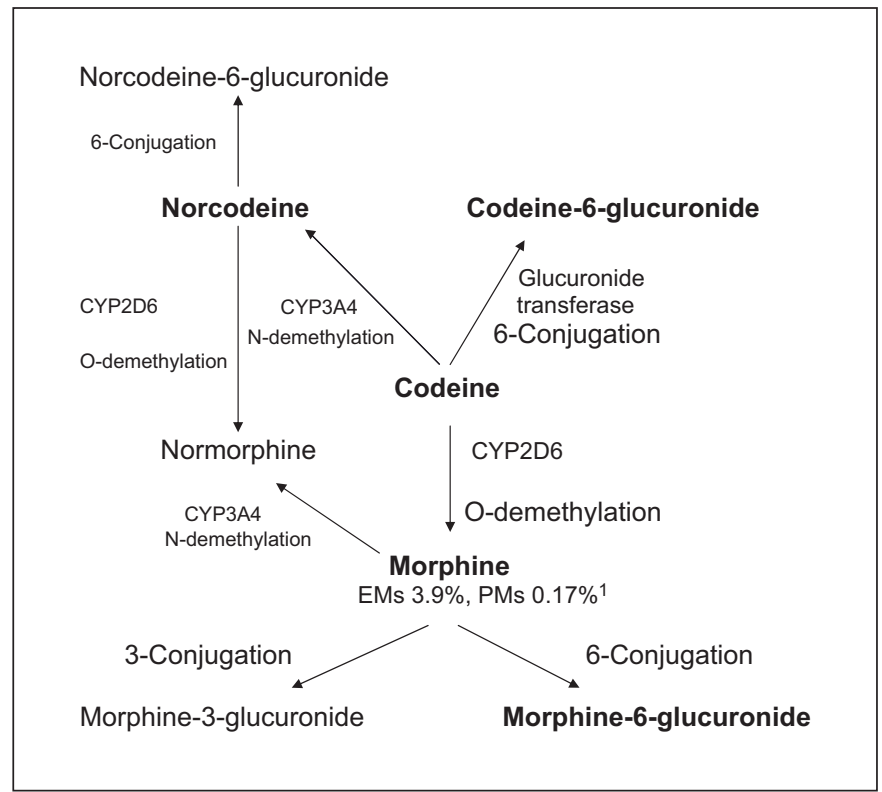

Fig. 3. Codeine metabolism, adapted from Lötsch et al. [66] and Gasche et al., [69]. The main compounds responsible for codeine analgesia are marked in bold. ${ }^{1}$ This percentage refers to morphine and its metabolites. Percentages of a single oral codeine dose found in the urine in EMs and PMs are shown.

ministered a single 30-mg dose of oral codeine. Genotyping using the polymerase chain reaction was performed and pharmacokinetics was measured during $24 \mathrm{~h}$ after drug administration. Significant differences were found between the EM and UM groups in AUCs versus time curves of morphine with a median AUC of 11 (range 5-17) $\mu \mathrm{g} \cdot \mathrm{h} \cdot \mathrm{liter}^{-1}$ in EMs and $16(10-24) \mu \mathrm{g} \cdot \mathrm{h} \cdot \mathrm{liter}^{-1}$ in UMs $(\mathrm{p}=0.02)$. The metabolic ratios in urine collected within $12 \mathrm{~h}$ after drug administration of codeine $+\mathrm{C}-6-\mathrm{G}$ divided by the sum of morphine + its glucuronide metabolites were 11 (range 6-17) in EMs and 9 (range 6-16) in UMs $(\mathrm{p}=0.05)$. Ten of the 11 CYP2D6 UMs felt sedation compared to $6(50 \%)$ of the $12 \mathrm{EM}(\mathrm{p}=0.03)$. CYP2D6 genotypes predicting ultrarapid metabolism resulted in about $50 \%$ higher plasma concentrations of morphine and its glucuronides compared with the EMs. No serious adverse events were observed probably because only a single low dose of codeine was administered.

Eckhardt et al. [71] conducted a randomized, placebocontrolled, double-blind study of analgesia with a single oral dose of $170 \mathrm{mg}$ codeine compared to a single oral dose of $20 \mathrm{mg}$ morphine and placebo in $9 \mathrm{EMs}$ and $9 \mathrm{PMs}$ using the cold pressor test. Compared to placebo, $20 \mathrm{mg}$ of morphine caused an increase in pain tolerance in both 
EM and PM phenotypes. Following administration of codeine, analgesia was observed only in EMs $(p<0.01)$ but not in PMs (not significant). Adverse events were more pronounced after morphine and codeine compared to placebo in both EMs and PMs. No differences between EMs and PMs regarding adverse effects of codeine were observed. Significant differences between EMs and PMs in the formation of morphine after codeine administration could be observed. Morphine plasma concentrations were similar in PMs and EMs after morphine administration. Following administration of codeine, morphine plasma concentrations were comparable to those after morphine administration only in EMs. In PMs, only traces of morphine were detected in plasma. The percentage of the codeine dose converted to morphine and its metabolites was 3.9\% in EMs and 0.17\% in PMs. Genetically determined interindividual variability in codeine analgesia is related to differences in morphine formation, which contributes to the codeine analgesia.

Sindrup et al. [72] evaluated the analgesic efficacy and kinetics of a single oral dose of $75 \mathrm{mg}$ codeine in $12 \mathrm{EMs}$ and 12 PMs in a double-blind, placebo-controlled, crossover study. Morphine could not be detected in the plasma of any of the PMs whereas detectable plasma levels were found in 10 of 12 EMs. Pain thresholds to laser stimuli were determined before codeine administration and 90 , 150 and $210 \mathrm{~min}$ after drug intake. Codeine significantly increased the pricking pain thresholds in the EMs ( $p<$ 0.05 ) with no significant changes in the PMs. No change in pain thresholds occurred with placebo in either EMs or PMs. In the EMs there was a significant positive correlation between the increase in pain threshold and the plasma concentration of codeine. The study supported the thesis that morphine formation contributes to codeine analgesia.

Sindrup et al. [73] studied the ability of quinidine to penetrate the blood-brain barrier and its possible impact on codeine O-demethylation in the CNS. 16 EMs and 1 $\mathrm{PM}$ underwent spinal anesthesia for urinary tract surgery or examination. Eight patients were given an oral dose of $125 \mathrm{mg}$ codeine and 9 patients including the PM were given $200 \mathrm{mg}$ quinidine $2 \mathrm{~h}$ before the administration of the same dose of codeine. Free concentrations of quinidine were 11-fold lower in CSF than in plasma. Morphine concentrations were significantly lower in patients pretreated with quinidine, both in plasma and in CSF. The morphine/codeine concentration ratio in plasma and in CSF was also lower. The morphine/codeine concentration ratios were significantly lower in CSF both without and with quinidine, but the difference between plasma and CSF ratios was significantly smaller with quinidine than without $(p=0.0002)$. Quinidine penetrates the blood-brain barrier poorly, but quinidine pretreatment leads to a pronounced lowering of the CSF concentration of morphine after codeine intake. The O-demethylation of codeine in the CNS may not be totally blocked by quinidine.

In a trial performed by Poulsen et al. [74], 14 EMs and 14 PMs completed a randomized, double-blind, threeway, cross-over study with a single oral dose of codeine (75 or $100 \mathrm{mg}$ ) against morphine (20 or $30 \mathrm{mg}$ ) and placebo. After morphine administration, morphine and M6-G were present in equal amounts in plasma of PMs and EMs. After codeine intake, neither morphine nor M-6-G could be detected in 13 of the $14 \mathrm{PMs}$ whereas at least one of the compounds could be detected in all EMs. Peak pain and discomfort during the cold pressor test were significantly reduced by morphine in both EM and PM. Codeine significantly reduced these pain measures only in EMs. Pain detection and tolerance thresholds to heat and pressure were not consistently altered by either morphine or codeine. In PMs, adverse effects were more pronounced after morphine than after codeine and only showed a slight difference between codeine and placebo. In EMs, there was no difference between codeine and morphine and more pronounced adverse effects of both drugs as compared to placebo. Codeine O-demethylation depends on CYP2D6; 6-glucuronidation of morphine is independent of CYP2D6. Codeine analgesia depends on its Odemethylation and this, probably, is also the case for adverse effects.

Yue at al. [75] compared the 8-hour urinary excretion of codeine and 7 of its metabolites in 149 healthy Swedish Caucasians and 133 healthy Chinese following a single oral dose of $25 \mathrm{mg}$ codeine phosphate. The Chinese were less able to metabolize codeine, in particular, by glucuronidation than the Caucasians. However, as observed with debrisoquine, the occurrence of genetically poor $\mathrm{O}$ demethylators of codeine was rare among the Chinese compared with the Caucasians.

Caraco et al. [76] compared the pharmacokinetics and pharmacodynamics of codeine with and without quinidine between Caucasian and Chinese EMs of debrisoquin. Codeine O-demethylation was significantly reduced after quinidine in both ethnic groups; however, the absolute decrease was greater in the Caucasians. The diminished production of morphine after quinidine was associated with a marked reduction in the effects of codeine $(p<0.01)$ in the Caucasians but not in the Chinese. The Chinese produce less morphine from codeine; they 
exhibit reduced sensitivity to that morphine, and therefore might experience a reduced analgesic effect in response to codeine. Quinidine-induced inhibition of codeine $\mathrm{O}$-demethylation is ethnically dependent with the reduction being greater in the Caucasians. Studies performed to date [69-77] indicate that codeine analgesia depends on CYP2D6 polymorphism and morphine and the formation of its glucuronides, but codeine and its metabolites (C-6-G and NORC) also contribute to the analgesic effects [66-68].

\section{Conclusions}

Experimental and clinical studies demonstrate that tramadol analgesia depends on CYP2D6 activity [46-49]. The studies performed to date [69-73] indicate that codeine analgesia also depends on CYP2D6 polymorphism but, apart from morphine and its metabolites, the parent compound and codeine metabolites, such as C-6-G and NORC, contribute to codeine analgesia [66-68]. Most studies $[56,59-61]$ indicate that DHC analgesia is independent of CYP2D6 activity [64] as, apart from O-demethylation to DHM, glucuronidation to DHC-6-G and $\mathrm{N}$-demethylation to NORDHC play an important role along with the parent compound activity [56]. As the codeine and DHC metabolic pathways are similar, the differences in analgesia with respect to CYP2D6 polymorphism may be explained by a stronger analgesic effect of the parent compound in the case of DHC [77]. DHC may be used as an alternative drug to tramadol when the latter is not tolerated (intense adverse effects such as nausea, sweating, dizziness and poor sensation of well-being) or provides inadequate analgesia. DHC is usually better tolerated than tramadol, rarely causes nausea and provides stronger analgesia. In contrast to tramadol, there seems to be little risk of triggering the serotonin syndrome when combining DHC with SSRIs.

\section{References}

1 Tucker G: Advances in understanding drug metabolism and its contribution to variability in patients' response. Ther Drug Monitor 2000;22:110-113.

$\checkmark 2$ Touw DJ: Clinical implications of genetic polymorphisms and drug interactions mediated by cytochrome P-450 enzymes. Drug Metabol Drug Interact 1997;14:55-82.

$\checkmark 3$ Wolf CR, Smith G: Cytochrome P450 CYP2D6. IARC Sci Publ 1999;148:209-229.

4 Ingelman-Sundberg M: Genetic polymorphism of cytochrome P450 2D6 (CYP2D6): clinical consequences, evolutionary aspects and functional diversity. Pharmacogenom J 2005;5:6-13.

5 Davis MP, Homsi J: The importance of cytochrome P450 monooxygenase CYP2D6 in palliative medicine. Support Care Cancer 2001;9:442-451.

6 Kroemer HK, Eichelbaum M: 'It's the genes, stupid'. Molecular bases and clinical consequences of genetic cytochrome P450 2D6 polymorphism. Life Sci 1995;56:2285-2298.

$\checkmark 7$ Stamer UM, Stüber F: Genetic factors in pain and its treatment. Curr Opin Anaesthesiol 2007;20:478-484.

8 Kostka-Trabka E, Woron J: Izoenzymy cytochromu P450 a problem interakcji lekow (Polish); in Kostka-Trabka E, Woron J (eds): Interakcje Lekow w Praktyce Klinicznej. Warszawa, Wydawnictwo Lekarskie PZWL, 2006, pp 13-24.

$\checkmark 9$ Daly AK, Brochmöller J, Broly F, et al: Nomenclature for human CYP2D6 alleles. Pharmacogenetics 1996;6:193-201.
10 Sachse C, Brochmöller J, Bauer S, Roots I: Cytochrome P450 2D6 Variants in a Caucasian population: allele frequencies and phenotyping consequences. Am J Hum Genet 1997;60:284-295.

11 Ingelman-Sundberg M, Oscarson M, McLellan RA: Polymorphic human cytochrome P450 enzymes: an opportunity for individualized drug treatment. Trends Pharmacol Sci 1999;20:342-349.

12 Raimundo S, Toscano C, Klein K, et al: A novel intronic mutation, $2988 \mathrm{G}>\mathrm{A}$, with high predictivity for impaired function of cytochrome P450 2D6 in white subjects. Clin Pharmacol Ther 2004;76:128-138.

$>13$ Masimirembwa CM, Hasler JA: Genetic polymorphism of drug metabolizing enzymes in African populations: implications for the use of neuroleptics and antidepressants. Brain Res Bull 1997;44:561-571.

14 Kirchheiner J, Keulen J-T HA, Bauer S, Roots I, Brockmöller J: Effects of the CYP2D6 gene duplication on the pharmacokinetics and pharmacodynamics of tramadol. J Clin Psychopharmacol 2008;28:78-83.

15 Wang G, Zhang H, He F, Fang X: Effect of the CYP2D6*10 C188T polymorphism on postoperative tramadol analgesia in a Chinese population. Eur J Clin Pharmacol 2006;62: 927-931.

16 Gan SH, Ismail R, Wan Adnan WA, Wan Z: Correlation of tramadol pharmacokinetics and CYP2D6 10 genotype in Malaysian subjects. J Pharm Biomed Anal 2002;30:189195.
17 Hennies MH, Friderichs E, Schneider J: Receptor binding, analgesic and antitussive potency of tramadol and other selected opioids. Arzneimittelforschung 1988;38:877-880.

-18 Raffa RB, Friderichs E, Reimann W, Shank RP, Codd EE, Vaught JL: Opioid and nonopioid components independently contribute to the mechanism of action of tramadol, an 'atypical' opioid analgesic. J Pharmacol Exp Ther 1992;260:275-285.

19 Raffa RB, Friderichs E, Reimann W, et al: Complementary and synergistic antinociceptive interaction between the enantiomers of tramadol. J Pharmacol Exp Ther 1993;267: 331-340.

20 Dickman A: Tramadol: a review of this atypical opioid. Eur J Palliat Care 2007;14:181-185.

21 Lintz W, Erlaçin S, Francus E, Uragg H: Biotransformation of tramadol in man and animal. Arznneimittelforschung 1981;31:19321943.

22 Wu WN, McKown LA, Liao S: Metabolism of the analgesic drug ULTRAM ${ }^{\circledR}$ (tramadol hydrochloride) in humans: API-MS and MS/ MS characterization of metabolites. Xenobiotica 2002;32:411-425.

23 Halling J, Weihe P, Brosen K: CYP2D6 Polymorphism in relation to tramadol metabolism: a study of Faroese patients. Ther Drug Monit 2008;30:271-275.

24 Subrahmanyam V, Renwick AB, Walters DG, et al: Identification of cytochrome P-450 isoforms responsible for cis-tramadol metabolism in human liver microsomes. Drug Metabol Disp 2001;29:1146-1155. 
25 Gillen G, Haurand M, Kobelt DJ, Wnendt S: Affinity, potency and efficacy of tramadol and its metabolites at the cloned human $\mu$ opioid receptor. Naunyn-Schmiedeberg's Arch Pharmacol 2000;362:116-121.

- 26 Valle M, Garrido MJ, Pavón JM, Calvo R, Trocóniz IF: Pharmacokinetic-pharmacodynamic modelling of the antinociceptive effects of main active metabolites of tramadol, (+)-O-desmethyltramadol and (-)-Odesmethyltramadol, in rats. J Pharmacol Exp Ther 2000;293:646-653.

27 Paar WD, Frankus P, Deugler HJ: The metabolism of tramadol by human liver microsomes. Clin Invest 1992;70:708-710.

28 Klotz U, Fischer-Bosch M: Tramadol - the impact of its pharmacokinetic and pharmacodynamic properties on the clinical management of pain. Arzneimittelforschung 2003;53:681-687.

-29 Mikus G, Somogyi AA, Bochner F, Chen ZR: Polymorphic metabolism of opioid narcotic drugs: possible clinical implications. Ann Acad Med Singapore 1991;20:9-12.

-30 Stamer UM, Lehnen K, Höthker F, Bayerer B, Wolf S, Hoeft A, Stuber F: Impact of CYP2D6 genotype on postoperative tramadol analgesia. Pain 2003;105:231-238.

- 31 Stamer U, Musshoff F, Kobilay M, Madea B, Hoeft A, Stuber F: Concentrations of tramadol and O-desmethyltramadol enantiomers in different CY2D6 genotypes. Clin Pharmacol Ther 2007;82:41-47.

- 32 Stamer U, Stuber F, Muders T, Musshoff F: Respiratory depression with tramadol in a patient with renal impairment and CYP2D6 gene duplication. Anesth Analg 2008;107: 926-929.

33 Garrido MJ, Sayar O, Segura C, et al: Pharmacokinetic/pharmacodynamic modelling of the antinociceptive effects of (+)-tramadol in the rat: role of cytochrome $\mathrm{P} 4502 \mathrm{D}$ activity. J Pharmacol Exp Ther 2003;305:710-718.

- 34 Paar WD, Poche S, Gerloff J, Dengler HJ: Polymorphic CYP2D6 mediates O-demethylation of the opioid analgesic tramadol. Eur J Clin Pharmacol 1997;53:235-239.

-35 Egberts ACG, Borgh JT, Brodie-Meijer CCE: Serotonin syndrome attributed to tramadol addition to paroxetine therapy. Int Clin Psychopharmacol 1997;12:181-182.

- 36 Mason BJ, Blackburn KH: Possible serotonin syndrome associated with tramadol and sertraline administration. Ann Pharmacother 1997;31:175-177.

37 Sauget D, Franco PS, Amaniou M, Mazere J, Dantoine T: Possible syndrome sérotoninergique induit par l'association de tramadol à de la sertraline chez une femme âgée. Thérapie 2002;57:309-310.

38 Davies MP: Tramadol; In Davies MP, Glare $\mathrm{P}, \mathrm{Hardy} J$ (eds): Opioids in Cancer Pain. Oxford, Oxford University Press, 2005, pp 6982.
39 Arcioni R, Rocca DM, Romano S, Romano R, Pietropaoli P, Gasparetto A: Ondansetron inhibits the analgesic effects of tramadol: a possible $5-\mathrm{HT}_{3}$ spinal receptor involvement in acute pain in humans. Anesth Analg 2002; 94:1533-1577.

40 Grond S, Sablotzki A: Clinical pharmacology of tramadol. Clin Pharmacokinet 2004; 43:879-923.

41 Gasse CBS, Derby L, Vasilakis-Scaramozza $\mathrm{C}$, Jick H: Incidence of first-time idiopathic seizures in users of tramadol. Pharmacotherapy 2000;20:629-634.

42 Rojas-Corrales MO, Ortega-Alvaro A Gibert-Rahola J, Roca-Vinardell A, Micó JA: Pindolol, a $\beta$-adrenoceptor blocker $/ 5$ hydroxytryptamine1A/1B antagonist, enhances the analgesic effect of tramadol. Pain 2000;88:119-124.

43 Sindrup SH, Madsen C, Brøsen K, Jensen TS: The effect of tramadol in painful polyneuropathy in relation to serum drug and metabolite levels. Clin Pharmacol Ther 1999;66: 636-641.

44 Poulsen L, Arendt-Nielsen L, Brøsen K, Sindrup SH: The hypoalgesic effect of tramadol in relation to CYP2D6. Clin Pharmacol Ther 1996;60:636-644.

45 Teppema LJ, Nieuwenhuijs D, Olievier CN, Dahan A: Respiratory depression by tramadol in the cat. Anesthesiology 2003;98:420427.

-46 Barnung SK, Treschow M, Borgbjerg FM: Respiratory depression following oral tramadol in a patient with impaired renal function. Pain 1997;71:111-112.

47 Mikus G, Weiss J: Influence of CYP2D6 genetics on opioid kinetics, metabolism and response. Curr Pharmacogenom 2005;3:4352.

48 Leppert W: Tramadol as an analgesic for mild to moderate cancer pain. Pharmacol Rep 2009;61:978-992.

49 Leppert W, Luczak J: The role of tramadol in cancer pain treatment - a review. Support Care Cancer 2005;13:5-17.

50 Gravenstein JS, Smith GM, Sphire RD, Isaacs JP, Beecher HK: Dihydrocodeine. N Engl J Med 1956;254:877-885.

51 Keats AS, Telford J, Kurosu Y: Studies of analgesic drugs: dihydrocodeine. J Pharmacol Exp Ther 1957;120:354-360.

52 Robertson JR: Dihydrocodeine: second strand of treatment for drug misusers. Drug Alcohol Rev 1996;15:200-201.

53 Ammon S, Hofmann U, Griese EU, Gugeler N, Mikus G: Pharmacokinetics of dihydrocodeine and its active metabolite after single and multiple oral dosing. Br J Clin Pharmacol 1999;48:317-322.

54 Rowell FJ, Seymour RA, Rawlins MD: Pharmacokinetics of intravenous and oral dihydrocodeine and its acid metabolites. Eur J Clin Pharmacol 1983;25:419-424.
55 Frazer NM, Galloway DB, Lockwood GF, McKeller J, Quinn KG: Single- and multipledose pharmacokinetic studies of DHC Continus tablets $60 \mathrm{mg}$, a novel dihydrocodeine preparation; in Doyle D (ed): 1986 Int Symp Pain Control. R Soc Med Serv Int Congr Symp Ser, No 123. London, Royal Society of Medicine Services, 1987, pp 101-107.

56 Schmidt H, Vormfelde SV, Walchner-Bonjean $\mathrm{M}$, et al: The role of active metabolites in dihydrocodeine effects. Int J Clin Pharmacol Ther 2003;41:95-106.

57 Fromm MF, Hofmann U, Griese EU, Mikus G: Dihydrocodeine: a new opioid substrate for the polymorphic CYP2D6 in humans. Clin Pharmacol Ther 1995;58:374-382.

58 Schmidt H, Vormfelde SV, Klinder K, et al: Affinities of dihydrocodeine and its metabolites to opioid receptors. Pharmacol Toxicol 2002;91:57-63.

59 Webb JA, Rostami-Hodjegan A, AbdulManap R, Hofmann U, Mikus G, Kamali F: Contribution of dihydrocodeine and dihydromorphine to analgesia following dihydrocodeine administration in man: a PK-PD modelling analysis. Br J Clin Pharmacol 2001;52:35-43.

-60 Jurna I, Kömen W, Baldauf J, Fleischer W: Analgesia by dihydrocodeine is not due to formation of dihydromorphine: evidence from nociceptive activity in rat thalamus. J Pharmacol Exp Ther 1997;281:1164-1170.

61 Wilder-Smith CH, Hufschmid E, Thormann $\mathrm{W}$ : The visceral and somatic antinociceptive effects of dihydrocodeine and its metabolite, dihydromorphine. A cross-over study with extensive and quinidine-induced poor metabolizers. Br J Clin Pharmacol 1998;45:575581.

62 Kirkwood LC, Nation RL, Somogyi AA: Characterization of the human cytochrome P450 enzyme involved in the metabolism of dihydrocodeine. Br J Clin Pharmacol 1997; 44:549-555.

63 Kirkwood LC, Nation RL, Somogyi AA: Glucuronidation of dihydrocodeine by human liver microsomes and the effect of inhibitors. Clin Exp Pharmacol Physiol 1998; 25:266-270.

64 Armstrong SC, Cozza KL: Pharmacokinetic drug interactions of morphine, codeine, and their derivatives: theory and clinical reality, part II. Psychosomatics 2003;44:515-520.

-65 Moore RA, Collins L, Carroll D, McQuay H: Paracetamol with and without codeine in acute pain: a quantitative systematic review. Pain 1997;70:193-201.

66 Lötsch J, Skarke C, Schmidt H, Rohrbacher M, Hofmann U, Schwab M, Geisslinger G: Evidence for morphine-independent central nervous opioid effects after administration of codeine: contribution of other codeine metabolites. Clin Pharmacol Ther 2006;79: $35-48$. 
-67 Vree TB, van Dongen RTM, Koopman-Kimenai PM: Codeine analgesia is due to codeine-6-glucuronide, not morphine. Int J Clin Pract 2000;54:395-398.

68 Vree TB, Verwey-van Wissen CP: Pharmacokinetics and metabolism of codeine in humans. Biopharm Drug Disp 1992;13:445460.

69 Gasche Y, Daali Y, Fathi M, et al: Codeine intoxication associated with ultrarapid CYP2D6 metabolism. N Engl J Med 2004; 351:2827-2831.

70 Kirchheiner J, Schmidt H, Tzetkov M, Keulen JT, Lötsch J, Roots I, Brockmöller J: Pharmacokinetics of codeine and its metabolite morphine in ultra-rapid metabolizers due to CYP2D6 duplication. Pharmacogenomics J 2007;7:257-265.
71 Eckhardt K, Li S, Ammon S, Schänzle G, Mikus G, Eichelbaum M: Same incidence of adverse drug events after codeine administration irrespective of the genetically determined differences in morphine formation. Pain 1998;76:27-33.

72 Sindrup SH, Brøsen K, Bjerring P, et al: Codeine increases pain thresholds to copper vapor laser stimuli in extensive but not poor metabolizers of sparteine. Clin Pharmacol Ther 1991;49:686-693.

73 Sindrup SH, Hofmann U, Asmussen J, et al Impact of quinidine on plasma and cerebrospinal fluid concentrations of codeine and morphine after codeine intake. Eur J Clin Pharmacol 1996;49:503-509.

74 Poulsen L, Brøsen K, Arendt-Nielsen L, Gram LF, Elbæk K, Sindrup SH: Codeine and morphine in extensive and poor metabolizers of sparteine: pharmacokinetics, analgesic effect and side effects. Eur J Clin Pharmacol 1996;51:289-295.
75 Yue QY, Svensson JO, Alm C, Sjöqvist F, Säwe J: Interindividual and interethnic difference in the methylation and glucuronidation of codeine. Br J Clin Pharmacol 1989;28: 629-637.

76 Caraco Y, Sheller J, Wodd AJJ: Impact of ethnic origin and quinidine coadministration on codeine's disposition and pharmacodynamic effects. J Pharmacol Exp Ther 1999; 290:413-422.

-77 Thompson CM, Wojno H, Greiner E, May EL, Rice KC, Selley DE: Activation of G-proteins by morphine and codeine congeners: insights to the relevance of $\mathrm{O}$ - and $\mathrm{N}$-demethylated metabolites at $\mu$ - and $\delta$-opioid receptors. J Pharmacol Exp Ther 2004;308: 547-554. 